\title{
Viscaceae endémicas del Perú
}

Blanca León ${ }^{1,2}$

${ }^{1}$ Museo de Historia Natural, Av. Arenales 1256, Aptdo. 14-0434, Lima 14, Perú

2 Plant Resources Center, University of Texas at Austin, Austin TX 78712 EE.UU.

blanca.leon@mail.utexas.edu

\section{Resumen}

La familia Viscaceae es reconocida en el Perú por presentar dos géneros y 69 especies (Brako \& Zarucchi, 1993; Ulloa Ulloa et al., 2004), todos arbustos parásitos. En este trabajo reconocemos 23 especies endémicas, en los géneros Dendrophthora y Phoradendron. Los taxones endémicos han sido encontrados principalmente en las regiones Bosques Muy Húmedos Montanos y Mesoandina, entre los 2100 y $3600 \mathrm{~m}$ de altitud. Se aplicaron las categorías y criterios de la UICN a 18 especies. Dos especies endémicas se encuentran representadas dentro del Sistema Nacional de Áreas Naturales Protegidas por el Estado.

Palabras claves: Viscaceae, Dendrophthora, Phoradendron, Perú, endemismo, plantas endémicas.

\section{Abstract}

The Viscaceae are represented in Peru by two genera and 69 species (Brako \& Zarucchi, 1993; Ulloa Ulloa et al., 2004), all parasitic shrubs. Here we recognize 23 endemic species in the genera Dendrophthora and Phoradendron. These endemic species are found mainly in Very Humid Montane Forests and Mesoandean regions, between 2100 and $3600 \mathrm{~m}$ elevation. We applied IUCN categories and criteria to 18 species. Two endemic species have been recorded within Peru's protected areas system.

Keywords: Viscaceae, Dendrophthora, Phoradendron, Peru, endemism, endemic plants.

\section{Dendrophthora diffusa Kuijt}

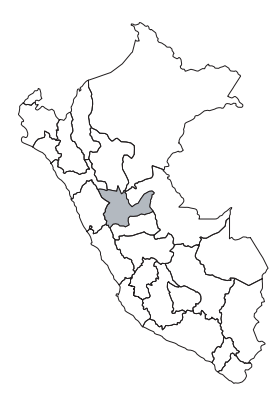
f. 3. 1987. m.

\section{CR, B1ab(iii)}

Publicación: Brittonia 39(4): 449—452,

Colección tipo: D.N. Smith et al. 2192

Herbarios: LEA, MO.

Nombre común: Desconocido.

Registro departamental: HU.

Regiones Ecológicas: MA; 2940—3100

SINANPE: Sin registro.

Herbarios peruanos: Ninguno.

Observaciones: Este arbusto parásito se conoce de una sola localidad, con fragmentos de bosque, donde fue registrada en 1982. Amenazas a sus poblaciones están asociadas a la deforestación, por ampliación de las actividades agrícolas.

\section{Dendrophthora dimorpha Kuijt}

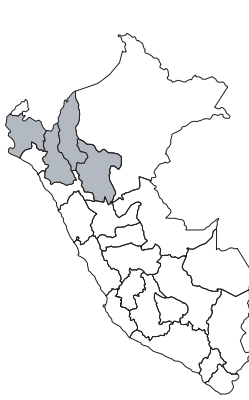

EN, B1ac(iii)

Publicación: Proc. Kon. Ned. Akad. Wetensch., Biol. 93(2): 132-134, f. 10. 1990.

Colección tipo: K.R. Young 2770

Herbarios: F; HUT.

Nombre común: Desconocido,

Registro departamental: AM, CA, SM. Regiones Ecológicas: BMHM; 2400$3250 \mathrm{~m}$.

SINANPE: PNC, PNRA

Herbarios peruanos: HUT (isotipo+2).

Observaciones: Arbusto parásito que se conoce del norte del país; las localidades extremas distan algo más de $200 \mathrm{~km}$ entre ellas. Se conoce de dos áreas protegidas, aunque la población en una de ellas, Parque Nacional Cutervo, puede estar afectada por la deforestación que ocurre en la zona.

\section{Dendrophthora fasciculata Pacz.}

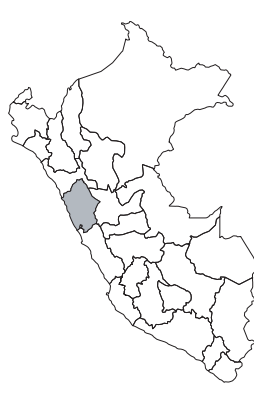

EN, B1a

Publicación: Bot. Jahrb. Syst. 45(4): 441. 1911.

Colección tipo: A. Weberbauer 3300

Herbarios: B(d); MOL!

Nombre común: Desconocido.

Registro departamental: AN.

Regiones Ecológicas: MA; 2700—2800

SINANPE: Sin registro.

Herbarios peruanos: MOL (isotipo).

Observaciones: Arbusto parásito, conocido aparentemente sólo de la colección tipo, una planta recolectada en matorrales xéricos del Callejón de Conchucos. Esta parte de la cuenca del Marañón requiere de estudios florísticos detallados.

\section{Dendrophthora ferruginea Pacz.}

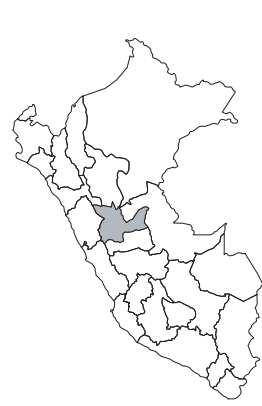

\section{EN, B1a}

Publicación: Bot. Jahrb. Syst. 45(4): 441. 1911.

Colección tipo: A. Weberbauer 3382

Herbarios: B; MOL!

Nombre común: Desconocido.

Registro departamental: HU.

Regiones Ecológicas: MA; 3300—3600

SINANPE: Sin registro.

Herbarios peruanos: MOL (isotipo), USM (1).

Observaciones: Arbusto parásito, conocido del dentro del país. El ejemplar tipo fue recolectado en la cuenca alta del Monzón, en 1903. Esta cuenca alberga varios endemismos, pero no ha sido herborizada en detalle desde la década de 1950. 


\section{Dendrophthora hexasticha Tiegh.}

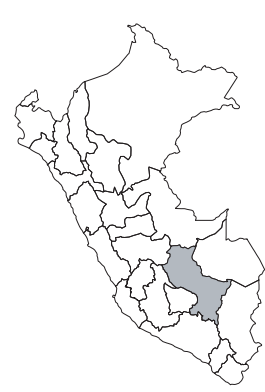

\section{NE}

Publicación: Bull. Soc. Bot. France 43: 181. 1896.

Colección tipo: J. Dombey 576

Herbarios: P.

Nombre común: Desconocido.

Registro departamental: CU.

Regiones Ecológicas: Sin datos; altitud desconocida.

SINANPE: Sin registro

Herbarios peruanos: Ninguno.

Observaciones: Arbusto parásito, conocido aparentemente de dos localidades. El ejemplar tipo fue recolectado en el siglo XVIII y se desconoce su procedencia.

\section{Dendrophthora linearifolia Pacz.}

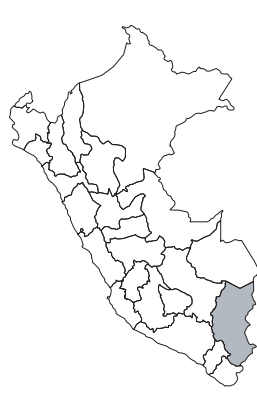

\section{DD}

Publicación: Bot. Jahrb. Syst. 45(4): 440. 1911.

Colección tipo: A. Weberbauer 539

Herbarios: B.

Nombre común: Desconocido.

Registro departamental: PU.

Regiones Ecológicas: BMHM; 2100$2300 \mathrm{~m}$.

SINANPE: Sin registro.

Herbarios peruanos: Ninguno.

Observaciones: Arbusto parásito, conocido del ejemplar tipo, recolectado en el sur del país a inicios del siglo XX. No se tiene datos de las características del hábitat de esta especie, aunque se presume que proviene del ecotono del bosque húmedo montano con el pluvial montano.

\section{Dendrophthora macbridei (Standl. ex J.F. Macbr.) Kuijt}

\section{DD}

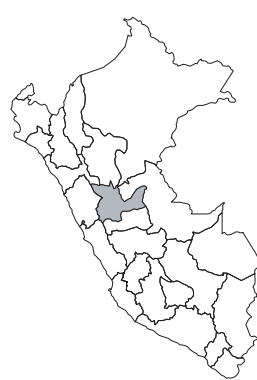

Publicación: Bot. Jahrb. Syst. 122(2): 177. 2000.

Colección tipo: J.F. Macbride 3954

Herbarios: F, NY.

Nombre común: Desconocido.

Registro departamental: HU.

Regiones Ecológicas: MA; $2100 \mathrm{~m}$.

SINANPE: Sin registro.

Herbarios peruanos: Ninguno.

Observaciones: Arbusto parásito conocido de la cuenca alta del Huallaga, la cual ha sido escasamente herborizada. Se desconoce el estado de sus poblaciones.

\section{Dendrophthora negeriana Pacz.}

\section{DD}

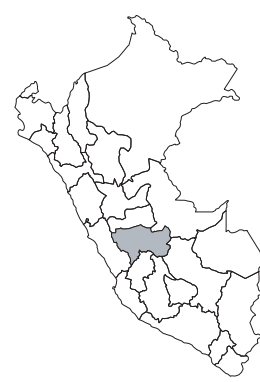

Publicación: Bot. Jahrb. Syst. 45(4): 440. 1911.

Colección tipo: A. Weberbauer 2138

Herbarios:

Nombre común: Desconocido.

Registro departamental: JU.

Regiones Ecológicas: BMHM; 2500$2600 \mathrm{~m}$.

SINANPE: Sin registro.

Herbarios peruanos: Ninguno.
Observaciones: Especie arbustiva, parásita, conocida de la localidad original, en la cuenca del Tulumayo. Fue descrita de una planta recolectada en 1903. Se desconoce el estado de sus poblaciones.

\section{Dendrophthora nodosa Pacz.}

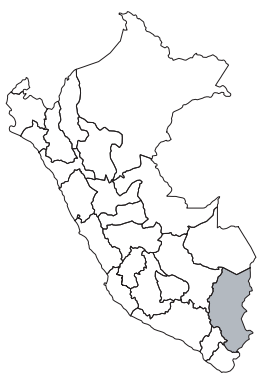

Publicación: Bot. Jahrb. Syst. 45(4): 439440. 1911.

Colección tipo: A. Weberbauer 1078

Herbarios: B (d).

Nombre común: Desconocido.

Registro departamental: PU.

Regiones Ecológicas: BMHM; 1800$2600 \mathrm{~m}$.

SINANPE: Sin registro.

Herbarios peruanos: Ninguno.

Observaciones: Este taxón fue considerado por Brako \& Zarucchi (1993) como un endemismo; sin embargo, no ha sido posible evaluarlo, ni asignarle una categoría.

\section{Dendrophthora pavoni Tiegh.}

Publicación: Bull. Soc. Bot. France 43: 182. 1896

Colección tipo: J. Pavón s.n.

Herbarios: G.

Nombre común: Desconocido.

Registro departamental: Sin datos.

Regiones Ecológicas: Sin datos; altitud desconocida.

SINANPE: Sin registro.

Herbarios peruanos: Ninguno.

Observaciones: Este taxón fue considerado por Brako \& Zarucchi (1993) como un endemismo; sin embargo, no ha sido posible evaluarlo, ni asignarle una categoría.

\section{Dendrophthora peruviana Kuijt}

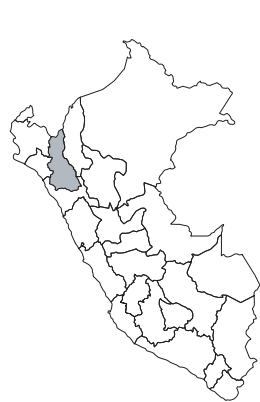

\section{DD}

Publicación: Wentia 6: 95-96, f. 42, pl. 11a. 1961.

Colección tipo: H.E. Stork \& O.B. Horton 10075

Herbarios: F, UC.

Nombre común: Desconocido.

Registro departamental: CA.

Regiones Ecológicas: MA; $2500-2800 \mathrm{~m}$.

SINANPE: Sin registro.

Herbarios peruanos: Ninguno.

Observaciones: Especie arbustiva, parásita, conocida de unas pocas localidades en el norte del país. Se desconoce el estado de sus poblaciones.

\section{Dendrophthora ramosa Pacz.}

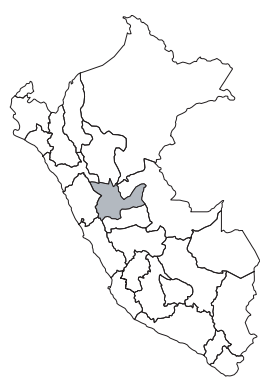

Publicación: Bot. Jahrb. Syst. 45(4): 440. 1911. Colección tipo: A. Weberbauer 3369

Herbarios: B(d); MOL!.

Nombre común: Desconocido.

Registro departamental: HU.

Regiones Ecológicas: BMHM; altitud desconocida.

SINANPE: Sin registro.

Herbarios peruanos: MOL (isotipo). 
Observaciones: Este taxón fue considerado por Brako \& Zarucchi (1993) como un endemismo; sin embargo, no ha sido posible evaluarlo, ni asignarle una categoría.

\section{Dendrophthora urbaniana Pacz.}

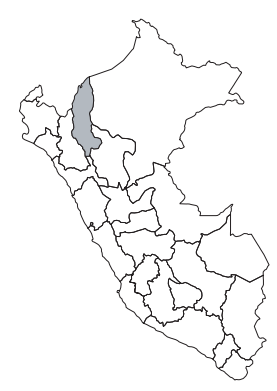

Publicación: Bot. Jahrb. Syst. 45(4): 440441. 1911.

Colección tipo: A. Weberbauer 4388

Herbarios: G; MOL!

Nombre común: Desconocido.

Registro departamental: AM.

Regiones Ecológicas: BMHM; 2400$2600 \mathrm{~m}$.

SINANPE: Sin registro.

Herbarios peruanos: MOL (isotipo).

Observaciones: Este taxón fue considerado por Brako \& Zarucchi (1993) como un endemismo; sin embargo, no ha sido posible evaluarlo, ni asignarle una categoría.

\section{Dendrophthora virgata (Trel. ex J.F. Macbr.) Kuijt}

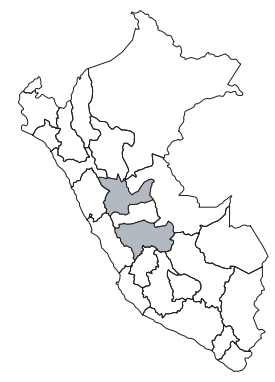

Publicación: Brittonia 32(4): 525, f. 10 11. 1980.

Colección tipo: E.P. Killip \& A.C. Smith 24350 Herbarios: F, NY.

Nombre común: Desconocido.

Registro departamental: HU, JU.

Regiones Ecológicas: BMHM; 2400$2800 \mathrm{~m}$.

SINANPE: Sin registro.

Herbarios peruanos: Ninguno.

Observaciones: Este taxón fue considerado por Brako \& Zarucchi (1993) como un endemismo; sin embargo, no ha sido posible evaluarlo, ni asignarle una categoría.

\section{Phoradendron carnosum Kuijt}

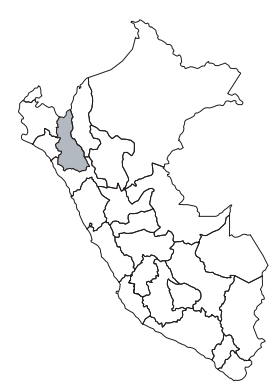

\section{DD}

Publicación: Syst. Bot. Monogr. 66: 156. 2003. Colección tipo: F. Woytkowski 5692 Herbarios: MO, US.

Nombre común: Desconocido.

Registro departamental: CA.

Regiones Ecológicas: BMHP; $1200 \mathrm{~m}$.

SINANPE: Sin registro.

Herbarios peruanos: Ninguno.

Observaciones: Arbusto parásito, conocido sólo de una localidad, probablemente en la Provincia de Jaén. Esta especie no ha vuelto a ser recolectada hace más de 50 años, además existe incertidumbre en cuanto a la localidad original y el hábitat de esta especie.

\section{Phoradendron grahamii Kuijt}

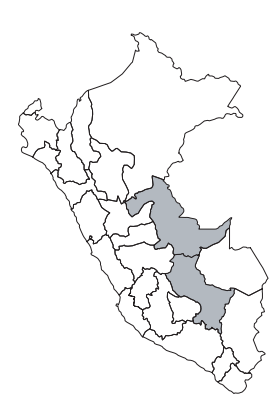

Observaciones: Arbusto parásito, conocido de dos localidades, en el centro y sur oriente del país. El ejemplar tipo fue recolectado en la cuenca del Ucayali, en 1998. Un ejemplar adicional proviene del área de influencia del gasoducto de Camisea.

\section{Phoradendron leucocarpum Pacz.}

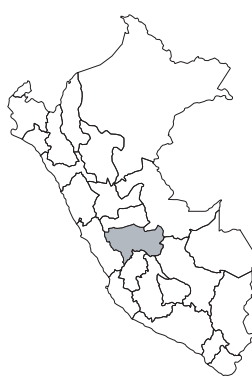

EN, B1a

Publicación: Bot. Jahrb. Syst. 45(4): 438439. 1911.

Colección tipo: A. Weberbauer 2193

Herbarios: B(d); MOL!.

Nombre común: Desconocido.

Registro departamental: JU.

Regiones Ecológicas: BMHM; 2500$2700 \mathrm{~m}$.

SINANPE: Sin registro.

Herbarios peruanos: MOL (s 2193).

Observaciones: Esta arbusto dioico y parásito se conoce de la localidad tipo, en la cuenca del Tulunayo. Probablemente, habita zonas ecotonales de bosque siempre húmedo con bosque estacionalmente seco.

\section{Phoradendron lingulatum Kuijt}

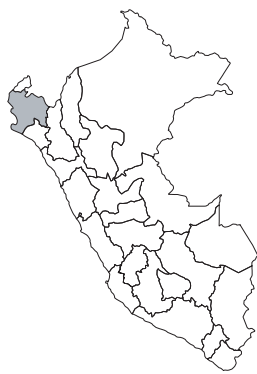

\section{CR, B1a}

Publicación: Syst. Bot. Monogr. 66: 275. 2003. Colección tipo: F. Woytkowski 6772a Herbarios: F, GH.

Nombre común: Desconocido. Registro departamental: PI.

Regiones Ecológicas: MA; $2400 \mathrm{~m}$. SINANPE: Sin registro.

Herbarios peruanos: Ninguno.

Observaciones: Esta especie arbustiva, dioica, se conoce solamente de una localidad, la cual está ubicada al sur-oeste de Piura, cerca al límite con el Departamento de Lambayeque. Esta es una localidad que alberga varios endemismos, la mayoría de los cuales, como para esta especie, fueron recolectados una sola vez antes de los 1970.

\section{Phoradendron loretoi Kuijt}

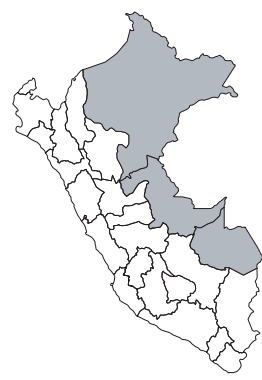
VU, B1a

Publicación: Syst. Bot. Monogr. 66: 282, f. 134, 169. 2003.

Colección tipo: C. Ramirez 85

Herbarios: LEA, MO, NY.

Nombre común: Desconocido.

Registro departamental: LO, MD, UC.

Regiones Ecológicas: BHA; 106-200 m. SINANPE: RNAM

Herbarios peruanos: Ninguno.

Observaciones: Arbusto parásito, conocido de cuatro localidades, en la Amazonía peruana. Una de las poblaciones en un área protegida, Reserva Nacional Allpahuayo-Mishana y otra en una reserva privada.

\section{Phoradendron mathewsii Trel.}

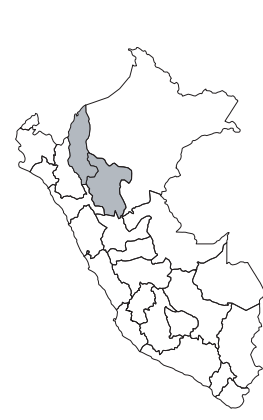

\section{NE}

Publicación: Phoradendron 133. 1916. Colección tipo: A. Mathews 3104 Herbarios: G, K.

Nombre común: Desconocido. Registro departamental: AM, SM. Regiones Ecológicas: BMHM; altitud desconocida.

SINANPE: Sin registro.

Herbarios peruanos: Ninguno. 
Observaciones: Arbusto parásito, conocido aparentemente, de dos localidades en el nor-oriente del país.

\section{Phoradendron neurophyllum Kuijt}

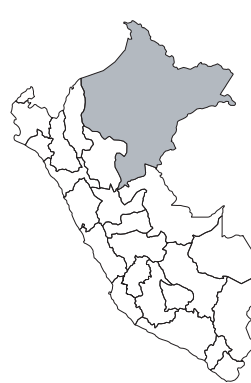

\section{DD}

Publicación: Syst. Bot. Monogr. 66: 317318. 2003.

Colección tipo: R. Vásquez \& N. Jaramillo 11595

Herbarios: LEA, MO.

Nombre común: Desconocido.

Registro departamental: LO.

Regiones Ecológicas: BHA; $110 \mathrm{~m}$.

SINANPE: Sin registro.

Herbarios peruanos: Ninguno.

Observaciones: Arbusto dioico, conocido de tres localidades, de la Amazonía peruana. Se desconoce el estado de sus poblaciones.

\section{Phoradendron storkii F.A. Barkley}

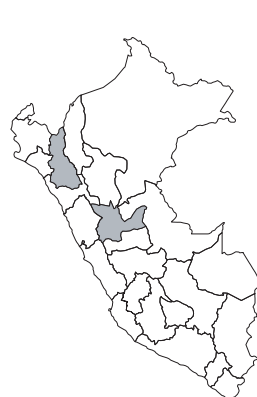

\section{EN, B1a}

Publicación: Madroño 9: 149-150, f. 21A. 1948.

Colección tipo: H.E. Stork \& O.B. Horton 10133

Herbarios: F.

Nombre común: Desconocido. Registro departamental: CA, HU.

Regiones Ecológicas: MA; $3200 \mathrm{~m}$.

SINANPE: Sin registro.

Herbarios peruanos: Ninguno.

Observaciones: Esta especie arbustiva, monoica, parásita, conocida de dos localidades en el norte del país. Ocupa probablemente ambientes ecotonales entre el bosque montano y matorrales mesoandinos. Este tipo de ambientes reciben escasa protección, especialmente en Cajamarca.

\section{Phoradendron vargasii Kuijt}

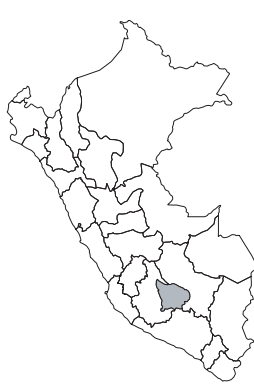

DD

Publicación: Syst. Bot. Monogr. 66: 470— 471, f. 298. 2003.

Colección tipo: C. Vargas C. 8853

Herbarios: $\mathrm{MO}$.

Nombre común: Desconocido.

Registro departamental: AP.

Regiones Ecológicas: MA; $2600 \mathrm{~m}$.

SINANPE: Sin registro.

Herbarios peruanos: Ninguno.

Observaciones: Esta especie arbustiva, parásita, se conoce solamente de una localidad en la cuenca del Apurímac. Aparentemente no ha vuelto a ser recolectado desde 1950. 